\title{
Sudden Vision Loss During Hemodialysis
}

Andrew W. Panakos, MD

Thomas Jefferson University, andrew.panakos@jefferson.edu

Follow this and additional works at: https://jdc.jefferson.edu/tmf

Part of the Medicine and Health Sciences Commons

Let us know how access to this document benefits you

\section{Recommended Citation}

Panakos, MD, Andrew W. (2015) "Sudden Vision Loss During Hemodialysis," The Medicine Forum: Vol. 16, Article 16.

DOI: https://doi.org/10.29046/TMF.016.1.015

Available at: https://jdc.jefferson.edu/tmf/vol16/iss1/16

This Article is brought to you for free and open access by the Jefferson Digital Commons. The Jefferson Digital Commons is a service of Thomas Jefferson University's Center for Teaching and Learning (CTL). The Commons is a showcase for Jefferson books and journals, peer-reviewed scholarly publications, unique historical collections from the University archives, and teaching tools. The Jefferson Digital Commons allows researchers and interested readers anywhere in the world to learn about and keep up to date with Jefferson scholarship. This article has been accepted for inclusion in The Medicine Forum by an authorized administrator of the Jefferson Digital Commons. For more information, please contact: JeffersonDigitalCommons@jefferson.edu. 


\title{
Sudden Vision Loss During Hemodialysis
}

\author{
Andrew W. Panakos, MD
}

\section{INTRODUCTION}

Catheter-related blood stream infections (CRBSIs) are a common and unfortunate consequence of prolonged vascular access in hemodialysis patients. Metastatic infections are feared sequelae of bacteremia, and include endocarditis, osteomyelitis, septic arthritis, epidural abscess, and endophthalmitis. ${ }^{1}$ The following is a case of Serratia endophthalmitis originating from a tunneled-dialysis catheter.

\section{CASE PRESENTATION}

A 47 year-old Caucasian female with a history of end stage renal disease on hemodialysis, Type 2 diabetes mellitus, and hypertension was undergoing her routinely scheduled hemodialysis session when she experienced "flashes of hot pink" in her left eye. The hemodialysis session was terminated, and the patient returned home. When she awoke the next morning she noticed a large black spot obscuring vision in her left eye as well as pain in the affected eye. The patient presented to Wills Eye Emergency Department that day. She denied any recent fevers, chills, pain, or erythema at the catheter site. She did, however, report that her left-sided chest wall tunneled dialysis catheter had been manipulated and tubing had been exchanged the day prior because of concerns that it was clogged. The patient was transferred to Thomas Jefferson University Hospital (TJUH) for further care.

Initial vital signs revealed a temperature of 98.6 degrees Fahrenheit, heart rate of 88 beats per minute, respiratory rate of 21 per minute, blood pressure of $160 / 94 \mathrm{mmHg}$, and $95 \%$ oxygen saturation on room air. Physical exam was significant for a diffusely erythematous left sclera with a hazy cornea and a small hypopion occupying the bottom third of the pupil (Figure 1). Her left pupil was sluggishly reactive to light. The patient's visual acuity exam of the left eye revealed 20/60 vision (20/20 in right eye). Her left chest wall port site was non-erythematous with no palpable fluctuance or drainage. There was no tenderness to palpation in this area. Cardiac exam revealed a grade II/VI systolic ejection murmur most prominent at the right upper sternal border. There were crackles at both lung bases and bilateral $2+$ lower extremity pitting edema to the shins. There were no splinter hemorrhages

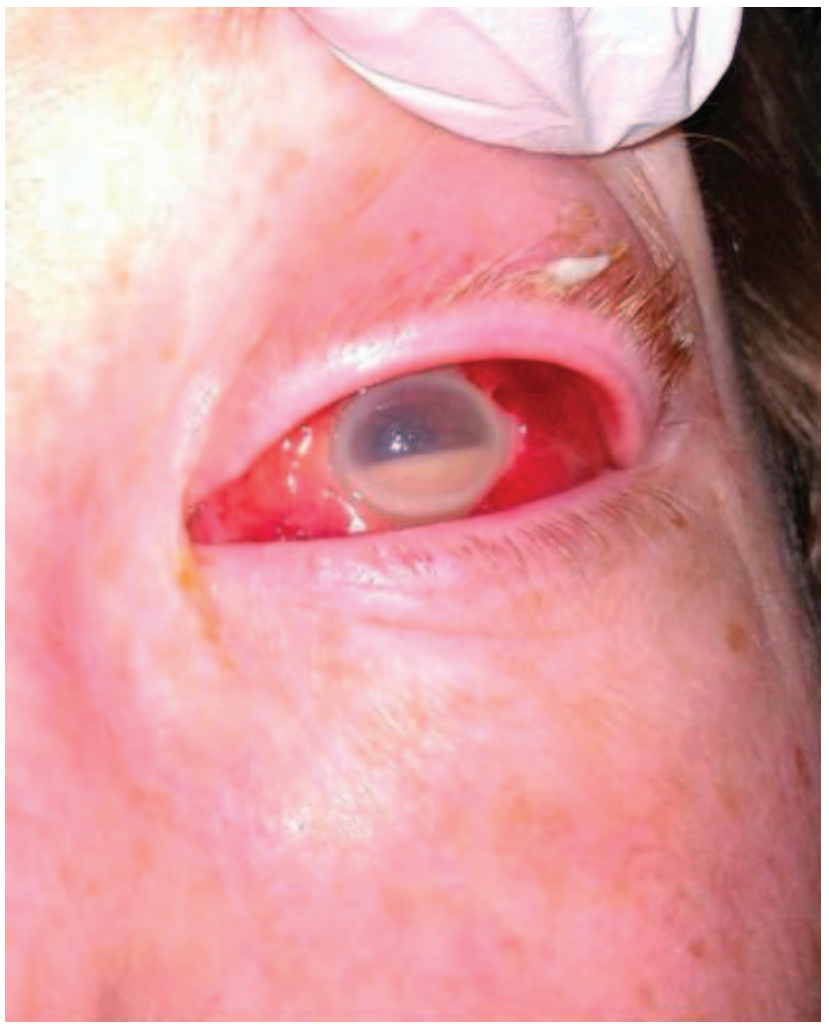

Figure 1. Patient's eye exam on day 3 of hospitalization, revealing significant hypopion and haziness of the cornea.

or other skin changes. The patient's admission laboratory values were notable for a white blood cell count of 16,200 cells/microliter (normal range 4,000-11,000) with 92\% neutrophils (normal range 40-73\%) and a hemoglobin of $7.5 \mathrm{~g} / \mathrm{dl}$ (normal 12.5-15) decreased from a baseline of $9 \mathrm{~g} / \mathrm{dl}$. A chest $x$-ray was normal.

\section{DIFFERENTIAL DIAGNOSIS}

The differential diagnosis for sudden vision loss is broad and can be divided into media disruptions, retinal problems, and neural visual pathway disturbances. Examples of media problems include keratitis, hyphema, lens changes, vitreous hemorrhage, uveitis, and endophthalmitis. Retinal pathology includes vascular occlusion, retinal detachment, and acute macular problems. Neural visual pathway disruptions can involve the optic nerve, chiasm, and retrochiasm. 


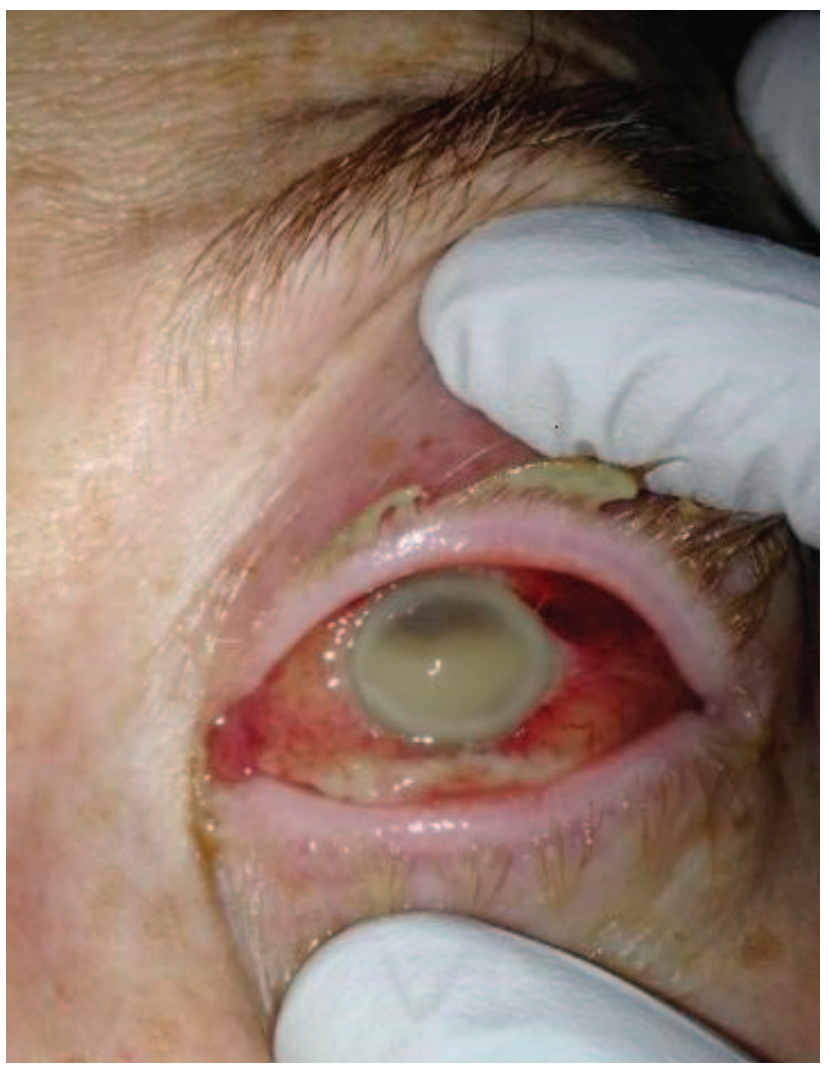

Figure 2. Patient's eye 7 days after admission. Notable are increased swelling, pus, worsening hypopion.

\section{OUTCOME AND FOLLOW-UP}

The patient's indwelling central venous access, elevated white blood cell count, and physical exam findings raised suspicion for an infectious cause of vision loss. A culture of the patient's vitreous fluid resulted as "many gram negative coccobacilli" five hours after being drawn, virtually clinching the diagnosis of bacterial endophthalmitis. The final culture revealed Serratia marcescens. Blood cultures drawn upon admission to TJUH grew gram negative bacilli one day after collection, and subsequently also speciated as Serratia marcescens. An ultrasound of the eye, also known as a "B scan", was performed by Ophthalmology and showed a large subretinal abscess and dense vitritis.

Her tunneled dialysis catheter was kept in place for 48 hours to allow her to receive two more dialysis sessions due to concern for refractory fluid overload. Subsequently, the catheter was removed and she was given a three day line holiday before replacement once her blood cultures had cleared. Of note, cultures from the catheter tip also grew gram negative bacilli.

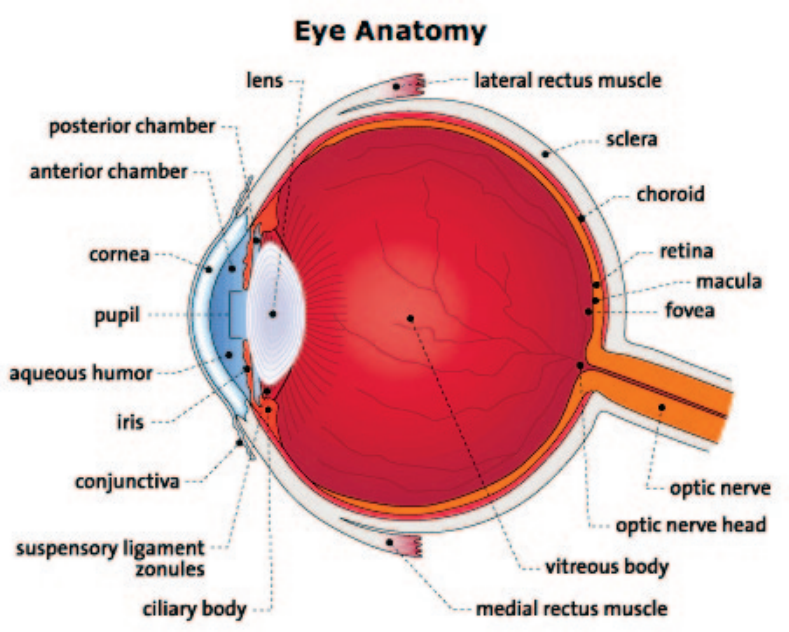

Figure 3. Structures of the eye. Endogenous endophthalmitis gains access to the eye through the choroid, the blood supply to the retina. http://www.harcourtcarteroptical.com/content2/anatomy

The patient was noted to have a systolic ejection murmur upon admission. It was unknown whether this was a new finding. Considering her evidence of metastatic infection, endocarditis was a concern (particularly involving the aortic valve). However, a transthoracic echocardiogram revealed no abnormalities involving the aortic valve or other vegetations. The patient's murmur resolved with red blood cell transfusion, thus it was felt that her initial murmur was a flow murmur related to anemia.

The patient was initially treated broadly with intraocular vancomycin, ceftazadime, moxifloxacin, and prednisolone, as well as intravenous vancomycin and piperacillintazobactam. Oral moxifloxacin was added on day 3 for its vitreal penetration. Intravitreal tobramycin and atropine eye drops were also added on day 5. Sensitivities returned and subsequently the patient's non-ocular antibiotics were de-escalated to only oral ciprofloxacin.

Despite these interventions, the patient suffered progressive vision loss and eye swelling (Figure 2). Oral prednisone was added but did not significantly improve her ocular symptoms. Ten days after presentation, the patient underwent enucleation of her left eye for progressive swelling, pain, and fear of refractory ocular infection which could spread beyond the orbit. An ocular implant was inserted during the same surgery. She was discharged on both oral and ocular ciprofloxacin as well as ocular moxifloxacin and a Medrol dose pack. 


\section{Panakos, MD: Sudden Vision Loss During Hemodialysis}

\section{DISCUSSION}

The most important modifiable risk factor for tunneled CRBSI is duration of catheter usage. ${ }^{3}$ One study found a $35 \%$ and $48 \%$ incidence of CRBSI at three and six months, respectively. ${ }^{3}$ The type of vascular access for hemodialysis patients also has an effect on the risk of infection. The relative risk of blood stream infection of a tunneled dialysis catheter compared with an arteriovenous fistula is 10:1. Additionally, the relative risk of a non-tunneled vs. tunneled dialysis catheter is $3: 1{ }^{3}$ Other risk factors for CRBSIs include previous CRBSI, recent surgery, diabetes mellitus, iron overload, immunosuppression, and hypoalbuminemia. ${ }^{3}$ Five techniques recommended by the Institute for Healthcare Improvement to prevent primary blood stream infections include effective hand hygiene, maximal barrier precautions at insertion, cutaneous antisepsis with chlorhexidine, optimal insertion site selection, and prompt removal when the catheter is no longer necessary. ${ }^{5}$ Microorganisms reach circulation in one of three ways: 1) colonization of insertion site (most common), 2) colonization of hubs, junctions, and other connectors-with subsequent access when these are manipulated, and 3) contamination of infused fluid. ${ }^{5}$

Endophthalmitis is a potential metastatic complication of CRBSIs. Endophthalmitis refers to a bacterial or fungal infection within the eye, including involvement of the vitreous and/or aqueous humors. ${ }^{4}$ Of note, endophthalmitis is not caused by viruses or parasites; eye infections due to these organisms are termed "uveitis". Endophthalmitis is further divided into exogenous and endogenous causes. Exogenous endophalmitis refers to infections that occur due to external factors such as trauma, surgery, or corneal infection. These account for the majority of cases of endophthalmitis. In contrast, endogenous endophthalmitis occurs due to seeding of the eye from within the body, typically through the choroid, which supplies blood to the retina.

\section{KEY POINTS}

Catheter related blood stream infections are a common and often avoidable occurrence. The incidence of CRBSIs in end stage renal disease patients is directly related to the amount of time the catheter is left in place. CRBSIs should be suspected in any patient with central venous access who presents with fevers/ chills or any signs of metastatic infection. Endogenous endophthalmitis is a potential metastatic manifestation of a CRBSI. Urgent ophthalmic consultation, intraocular and systemic antibiotics/antifungals, as well as catheter removal are the mainstays of treatment.

\section{REFERENCES}

1. Allon M. Dialysis catheter-related bacteremia: treatment and prophylaxis. American Journal of Kidney Disorders 2004; 44:779.

2. Leveque T, Tobe J, Park L. Approach to the adult with acute persistent vision loss. Up to Date January 11, 2014. http://www. uptodate.com/contents/approach-to-the-adult-with-acutepersistent-visual-loss

3. Allon M, Sexton D, Berns J, et al. Tunneled, cuffed hemodialysis catheter-related bacteremia. Up to Date January 11, 2014. http:// www.uptodate.com/contents/tunneled-cuffed-hemodialysiscatheter-related-bacteremia

4. Lee T, Barker J, Allon M. Tunneled catheters in hemodialysis patients: reasons and subsequent outcomes. American Journal of Kidney Disorders 2005; 46:501

5. Goldman L, Schafer A. Goldman's Cecil Medicine. 24th Edition. Elsevier. Pages 1785-1786.

6. Mermel L, Allon M, Bouza E, et al. Clinical Practice Guidelines for the Diagnosis and Management of Intravascular CatheterRelated Infection: 2009 Update by the Infectious Disease Society of America

7. Okada AA, Johnson RP, Liles WC, et al. Endogenous bacterial endophthalmitis: report of a ten-year retrospective study. Ophthalmology. 1994;101(5):832-838

8. Darouiche, RO, Wall MJ, Itani KM, et al. Chlorhexidine-alcohol versus povidone-iodine for surgical site antisepsis. N Engl J Med. 2010 Jan 7;362(1):18-26

9. Michiel G. H. Betjes, MD. Prevention of Catheter-related blood stream infections in patients on hemodialysis. Medscape. January 11, 2014. http://www.medscape.org/viewarticle/739174 